\title{
Neuropsychological profiles of adults and older adults with HIV
}

\author{
N Cassimjee* and P.K Motswai** \\ Corresponding author: \\ *Nafisa Cassimjee, Department of Psychology, University of Pretoria, Lynnwood Road, Pretoria, 0001. \\ Email:nafisa.cassimjee@up.ac.za. \\ ** Phenyo Motswai, Department of Psychology, University of Pretoria
}

\begin{abstract}
The wider availability of highly active anti-retroviral therapy has resulted in a concomitant increase in adults aging with HIV and the persistence of milder forms of neuropsychological impairment in this cohort. This study investigated the differences in neuropsychological functioning between a group of HIV positive $(H I V+)$ adults and older adults and an HIV negative (HIV-) matched control group. Participants from a semiurban community clinic volunteered to participate in the study. The performance of fifty participants (33 HIV+ and $17 \mathrm{HIV}-)$ who met the inclusion criteria were compared on the following measures: Dementia Rating Scale-2, the Stroop Colour and Word Test, the Symbol Digits Modalities Test and the D-KEFS Trail Making Test. The results indicated that the HIV positive in comparison to the HIV negative group had poorer performance profiles in global cognitive functioning, memory, executive functioning, visuoconstruction ability, psychomotor functioning and processing speed. The findings suggest that further research in South Africa will contribute to a better understanding of the
\end{abstract}


neuropsychological profiles of adults aging with HIV and inform intervention strategies specific to addressing the mental health care needs of this subgroup.

\section{Keywords}

Aging, Dementia Rating Scale-2, HIV, neuropsychological functioning, older adults, South Africa.

Due to the resource challenged context in Africa, particularly in the health care sector, few envisaged or planned for a surviving aging African population with Human Immunodeficiency Virus (HIV) (Mills, Rammohan, \& Awofeso, 2011). This scenario is commensurate with the limited number of studies in Africa that have focused on aging with HIV and the increasing risk for cognitive impairment in this cohort. Research on the neuropsychological functioning in this group is motivated by the demographic shift from primarily young individuals (91\% below 45 years of age in 1996) to approximately one half of the HIV seropositive adults currently over 45 years of age (Wendelken \& Valcour, 2012). The South African scenario parallels those observed internationally with the aging HIV population expected to increase by approximately $50 \%$ in the next 15 years (Hontelez et al., 2011).

Notwithstanding the success of highly active anti-retroviral therapy (HAART), the long term use of anti-retroviral drugs and their consequent central nervous system neurotoxicity may result in compromised cognitive integrity in an aging brain more susceptible to the neuropathological dynamics of HIV infection (Gannon, Khan, \& Kolson, 2011; Liner, Ro, \& Robertson, 2010). Baseline cerebral blood flow in HIV positive (HIV+) individuals was found to be equivalent to that of HIV negative (HIV-) 
individuals 15-20 years older (Ances et al., 2010). In an adult and older adult HIV+ cohort, Fazeli et al. (2014) elucidated both the presence of subtle cognitive impairment in $47 \%$ of the study sample and the persistence of a mild and diffuse pattern of cognitive impairment in the era of HAART.

Recent cross-sectional and longitudinal studies and reviews have explicated the differences in neuropsychological profiles between HIV+ and HIV- adults and older adults, with lower performance on measures of psychomotor functioning, attention, processing speed, executive functioning and memory evident in the HIV+ group (Cysique, Maruff, Bain, Wright, \& Brew, 2011; Seider et al., 2014; Woods, Moore, Weber, \& Grant, 2009). Furthermore, a comparative study revealed that HIV+ older individuals evidenced greater dispersion in neuropsychological performance across cognitive domains than HIV- individuals. The authors concluded that this is likely a function of compromised fronto-striatal systems that predispose the older HIV+ cohort to dysregulation of executive control (Morgan et al., 2011). Within the memory domain, for example, deficits observed in older HIV+ individuals may be indicative of compromised executive functioning such as flawed utilization of clustering strategies (Iudicello, Woods, Deutsch, Grant, \& The HIV Neurobehavioral Research Program Group, 2012).

Equivocal findings in the literature relating to the influence of age and HIV status on cognitive functioning have been reported. Both cross-sectional and longitudinal studies have found independent and synergistic effects and a recent study showed no independent or synergistic influences of age and serostatus on incident cognitive disorders (Scott et al., 2011; Seider et al., 2014; Sheppard et al., 2015). However, cognitive performance profiles consistently show poorer outcomes for the older cohorts 
with HIV (Vance, Fazeli, \& Gakumo, 2013). A review of studies using a screening assessment (International HIV-Dementia Scale) and conducted specifically in SubSaharan Africa yielded evidence that HIV is a major predisposing factor for cognitive impairment, with a frequency of approximately $40 \%$ in HIV+ patients on anti-retroviral therapy (Habib et al., 2013). Meta-analyses indicated that no significant association between age and cognitive impairment was evident. Utilizing a comprehensive neuropsychological battery, Lawler et al. (2011) found that $37 \%$ of HIV+ patients showed deficits in all cognitive and motor domains (memory, exeutive functioning, psychomotor speed, language, information processing speed and capacity and fine motor ability) when compared to matched uninfected control participants. Moreover, among the HIV+ group, those who evidenced cognitive impairment were older and had lower education levels than the cognitively unimpaired participants.

Until recently, cognitive status has been exclusively studied in developed countries on younger cohorts with HIV, and in genotypically defined subtypes specific to these regions. In the era of HAART with the commensurate extension of life expectancy, the trajectory of HIV is transforming from an acute to a more chronic inflammatory disease process and the prevalence and profile of cognitive deficits may reflect this change (Wendelken \& Valcour, 2012). There are limited studies on the aging population with HIV in South Africa and the changing disease parameters indicate that cognitive functioning in this subgroup remains comparatively understudied when compared to younger HIV+ cohorts. Based on the above exposition, the current study aims to explore the differences in neuropsychological outcomes between a group of HIV+ adults and older adults and an HIV- matched control group. 


\section{Method}

\section{Participants}

Eighty three (83) participants were recruited for the study over a period of 8 months; however 33 participants did not meet the minimum requirements as outlined in the exclusion criteria below. The remaining 50 eligible participants consisted of $33 \mathrm{HIV+}$ participants and 17 HIV- participants. A larger sample size could not be collected due to practical considerations such as time frame, cost, patient accessibility and incomplete medical information and specificity of the clinical sample. However, the sample size was still relevant to the preliminary and exploratory nature of the research (Hardy \& Vance, 2009). All participants included in the study were conversant in English and had English as a second language. Individuals were not eligible for participation if they had an unconfirmed HIV status according to medical records, a diagnosed psychiatric disorder, head injury with a loss of consciousness for more than 30 minutes, neurologic diseases, opportunistic brain infections, recent (within the last 3 months) history of substance abuse, cytomegalovirus (CMV) retinitis/other overt retinal conditions or ocular opportunistic infections and dementia.

\section{Instruments}

Due to limited context-specific norms in Sub-Saharan Africa (Singh et al., 2010), only the raw scores on the instruments were compared between the HIV+ and HIV- groups. In essence, no formal diagnoses or classifications were made, only preliminary comparisons were made in this clinical population. The following instruments were used to assess participants. 
The Dementia Rating Scale-2 (DRS-2) (Jurica, Leitten, \& Mattis, 2001); which has 36 tasks which comprise five subscales. These subscales include attention (DRS-2 Attention), initiation/perseveration (DRS-2 Initiation/Perseveration), construction (DRS2 Construction), conceptualisation (DRS-2 Conceptualisation), memory (DRS-2 Memory) and a global neuropsychological score (DRS-2 Total). Internal consistency and alpha coefficients of 0.95 (Attention), 0.87 (Initiation/Perseveration), 0.95 (Conceptualisation) and 0.75 (Memory) were reported (Vitaliano et al., 1984). The DRS2 is reported to be sensitive to cognitive impairment that has a subcortical profile and this test has been utilized in preceding HIV studies (Antinori et al., 2007, Suarez et al., 2000).

The Stroop Colour and Word Test (Stroop), the Symbol Digits Modalities Test (SDMT) and the Delis-Kaplan Executive Function System (D-KEFS) - Trail Making Test (TMT) were included as additional neuropsychological measures. The D-KEFS TMT comprises five conditions and for the purposes of this study the results of the D-KEFS Visual Scan (condition 1), the D-KEFS-Task Switching (condition 4) and the D-KEFSMotor Speed (condition 5) were reported. Test descriptions and psychometric properties are reported in Lezak, Howieson, Bigler, and Tranel (2012).

The health-related quality of life questionnaire (EQ-5D) - a standardised measure of general health-related quality of life was used to assess self-reported mood (anxiety/depression) (Robberstad \& Olsen, 2010). A socio-demographic questionnaire was used to gather information on age, gender, employment status and education. Clinical information was obtained from patients' medical records. 


\section{Procedure}

Potential participants were recruited from a government run community clinic with an HIV wellness centre. The clinic was located in a semi-urban area of Tshwane. The HIV+ group consisted of individuals who had been consecutively enrolled during routine outpatient visits. The control group consisted of HIV- individuals attending the community clinic in their capacity as caregivers, family members and other community dwelling volunteers. Individuals identified by the medical staff as meeting the inclusion criteria of the study were given a pamphlet explaining the basic purpose of the research study (in English, Afrikaans and Tswana). The pamphlets contained a tear-off slip on which interested participants could provide their contact details and place it in a box located in the examination room of the clinic.

Potential participants were contacted telephonically in order to set up an appointment. Participants who met the eligibility criteria and agreed to participate formalized their intention to partake in the study by signing informed consent forms and this was followed by a more comprehensive explanation of what was required of them. All measures were administered in English by two trained masters psychology students (clinical and research) under the supervision of a qualified psychologist in the neuropsychology field. The assessment session spanned approximately 30 minutes to an hour, depending on the pace of the participant. 


\section{Ethical Considerations}

Ethical approval to conduct the study was granted by the Humanities Postgraduate and Research Ethics Committee of the University of Pretoria and the Department of Health (Tshwane) Research Committee.

\section{Data Analysis}

Statistical analysis was conducted using the Statistical Analysis Software (SAS) and the Statistical Package for the Social Sciences (SPSS) version 21. Crosstabs and MannWhitney $U$ tests were conducted to summarize the data and evaluate socio-demographic comparability. The distribution of the neuropsychological performance scores was evaluated by means of the Kolmogorov-Smirnov test, skewness and kurtosis (Pallant, 2010). Due to the assumption of normality within the distributions not being met (as is common in clinical data); the nonparametric alterative to the t-test (the Mann-Whitney U test) was used to compare neuropsychological performance between the groups (Field, 2009). Due to the small sample size of the control group $(n=17)$, an additional nonparametric technique called the bootstrap resampling method was used (Chernick \& LaBudde, 2011). Spearman's rank-order correlation analysis was used to determine associations between clinical variables and neuropsychological performance in the HIV+ group. 
Table 1. Demographic characteristics and Chi-square statistics by HIV status

\begin{tabular}{|c|c|c|c|c|c|c|c|}
\hline Variable & HIV+ & & HIV- & & $X^{2}$ & $p$ & phi \\
\hline & Female & Male & Female & Male & \multirow{3}{*}{54.56} & \multirow{3}{*}{$<.000$} & \multirow{3}{*}{1.005} \\
\hline \multirow[t]{2}{*}{ Gender $\%$} & 67 & 33 & 76 & 24 & & & \\
\hline & Employed & Unemployed & Employed & Unemployed & & & \\
\hline \multirow[t]{2}{*}{ Employment \% } & 52 & 48 & 53 & 47 & \multirow[t]{2}{*}{.000} & \multirow[t]{2}{*}{.995} & \multirow[t]{2}{*}{.044} \\
\hline & Black & Other & Black & Other & & & \\
\hline Ethnicity \% & 100 & 0 & 100 & 0 & & & \\
\hline
\end{tabular}

Note: Sig level at $\mathrm{p}<.05, \mathrm{n}=50, X^{2}=$ chi-square statistic

\section{Results}

\section{Socio-demographic Variables}

Socio-demographic variables (gender, employment, and ethnicity) are summarised in Table 1. Chi-square tests for contingency indicated a significant association between HIV and gender $\left(\mathrm{X}^{2}(1, \mathrm{n}=50)=54.56, p<.001, p h i=1.005\right)$; however no significant association $\left(\mathrm{X}^{2}(1, \mathrm{n}=50)=.000, p=.995, p h i=.044\right)$ was observed between HIV status and employment status. Comparisons using Mann-Whitney U tests (Table 2) indicated no significant differences between the two groups with regard to years of education ( $p$ $=.076)$ and age $(\mathrm{p}=.169)$. Groups were also comparable with regard to anxiety/depression scores, with no significant differences $(\mathrm{p}=.767)$ in self-reported mood states. 
Table 2. Descriptives and Mann-Whitney statistics for demographic characteristics

\begin{tabular}{|c|c|c|c|c|c|c|c|c|}
\hline \multirow[t]{2}{*}{ Variable } & \multicolumn{3}{|l|}{$\mathrm{HIV}+$} & \multicolumn{5}{|l|}{ HIV- } \\
\hline & Mean & SD & Median & Mean & SD & Median & $\mathrm{U}$ & $\mathrm{p}$-value \\
\hline Education & 9.82 & 3.07 & 10 & 11.53 & 2.38 & 12 & 196.50 & .076 \\
\hline Age & 49.64 & 4.54 & 49 & 52.18 & 5.92 & 53 & 213.50 & .169 \\
\hline Anx/Deppr. & 1.62 & 0.99 & 1 & 2.06 & 1.00 & 1 & 268.00 & .767 \\
\hline
\end{tabular}

Note: Sig level at $\mathrm{p}<.05 ; \mathrm{n}=50 ; \mathrm{SD}=$ standard deviation; $\mathrm{U}=$ Mann-Whitney test statistic

\section{Clinical Variables}

Chronicity (determined by means of the duration between earliest dated HIV positive test result and the date of neuropsychological examination) showed a mean and median of 42.24 months $(\mathrm{SD}=37.55)$ and 31 months respectively. Duration on anti-retroviral medication (determined by means of the date given for anti-retroviral therapy initiation and the date of neuropsychological examination) showed a mean and median of 43.18 months ( $\mathrm{SD}=42.53)$ and 26 months respectively. The mean and median CD4 cell count was 415 cell $/ \mu \mathrm{L}(\mathrm{SD}=231.60)$ and 403 cell $/ \mu \mathrm{L}$ respectively, with 5 individuals with a CD4 cell count under 200 cell/ $\mu \mathrm{L}$.

Correlational analyses found a moderate inverse association between duration on anti-retroviral therapy and psychomotor processing $\left(r_{s}=-.33, p<.05\right)$. The number of months on anti-retroviral therapy (duration) also demonstrated a moderate, positive association with the D-KEFS measure of task-switching $\left(r_{s}=.40, p<.05\right)$. 
Table 3. Descriptive neuropsychological performance scores by HIV status

\begin{tabular}{|c|c|c|c|c|c|c|}
\hline & $\mathrm{HIV}+(\mathrm{r}$ & & & HIV- $(n$ & 17) & \\
\hline Variable & Mean & SD & Med & Mean & SD & Med \\
\hline DRS-Attention & 33.91 & 2.53 & 34 & 34.18 & 2.04 & 34 \\
\hline DRS-Initiation/Perseveration & 30.82 & 5.30 & 31 & 34.00 & 4.00 & 36 \\
\hline DRS-Construction & 5.85 & 0.36 & 6 & 5.88 & 0.33 & 6 \\
\hline DRS- Conceptualisation & 30.97 & 4.53 & 31 & 35.18 & 4.07 & 37 \\
\hline DRS-Memory & 22.55 & 2.46 & 23 & 23.65 & 1.80 & 24 \\
\hline DRS-Global & 124 & 11.37 & 125 & 132.29 & 9.51 & 132 \\
\hline SDMT-Psychomotor Processing & 26.00 & 9.49 & 27 & 32.19 & 9.85 & 32 \\
\hline STROOP-Selective Attention & 23.03 & 8.98 & 23 & 27.47 & 11.07 & 31 \\
\hline D-KEFS-Visual Scan & 42.54 & 17.75 & 39 & 32.75 & 9.90 & 31 \\
\hline D-KEFS-Switching & 200.20 & 54.45 & 240 & 173.81 & 68.97 & 180 \\
\hline D-KEFS-Motor Speed & 67.43 & 27.66 & 61 & 57.06 & 29.63 & 52.50 \\
\hline
\end{tabular}

$\mathrm{SD}=$ standard deviation; Med $=$ median

As shown in Table 4, the HIV+ group had poorer global neuropsychological performance in comparison to the HIV-group $(p<.00195 \%$ CIs $[0.006] \&[0.098])$. The executive function domain had the most statistical support for poorer performance by the HIV+ group in comparison to the HIV- group. This was evidenced by the following indicators: switching $(p=.027,95 \%$ CIs [0.138] \& [0.690]), initiation/perseveration $(p$ $<.001,95 \%$ CIs [0.004] \& [0.093]) and conceptualization $(\mathrm{p}<.001,95 \%$ CIs [0.002] \& [0.033]). 
Table 4. Comparative neuropsychological performance between the HIV+ and HIV-groups.

\begin{tabular}{|c|c|c|c|c|c|}
\hline \multirow[t]{2}{*}{ Neuropsychological Tests } & \multirow[t]{2}{*}{ Group } & \multirow[t]{2}{*}{ Median } & \multicolumn{2}{|c|}{$95 \% \mathrm{CI}$} & \multirow[t]{2}{*}{ p-value } \\
\hline & & & Lower & Upper & \\
\hline \multirow[t]{2}{*}{ DRS-Attention } & HIV+ & 34 & 0.328 & 0.810 & .065 \\
\hline & HIV- & 34 & & & \\
\hline \multirow[t]{2}{*}{ DRS-Initiation/Perseveration } & $\mathrm{HIV}+$ & 31 & 0.004 & 0.093 & $<.001$ \\
\hline & HIV- & 36 & & & \\
\hline \multirow[t]{2}{*}{ DRS-Construction } & $\mathrm{HIV}+$ & 6 & 0.732 & 0.950 & $<.001$ \\
\hline & HIV- & 6 & & & \\
\hline \multirow[t]{2}{*}{ DRS-Conceptualisation } & $\mathrm{HIV+}$ & 31 & 0.002 & 0.033 & $<.001$ \\
\hline & HIV- & 37 & & & \\
\hline \multirow[t]{2}{*}{ DRS-Memory } & $\mathrm{HIV}+$ & 23 & 0.424 & 0.518 & $<.001$ \\
\hline & HIV- & 24 & & & \\
\hline \multirow[t]{2}{*}{ DRS-Global } & HIV+ & 125 & 0.006 & 0.098 & $<.001$ \\
\hline & HIV- & 132 & & & \\
\hline \multirow[t]{2}{*}{ SDMT-Psychomotor Processing } & $\mathrm{HIV}+$ & 27 & 0.030 & 0.282 & $<.001$ \\
\hline & HIV- & 32 & & & \\
\hline \multirow[t]{2}{*}{ STROOP-Selective Attention } & $\mathrm{HIV}+$ & 23 & 0.104 & 0.670 & .052 \\
\hline & HIV- & 31 & & & \\
\hline \multirow[t]{2}{*}{ D-KEFS-Visual Scan } & $\mathrm{HIV+}$ & 39 & 0.071 & 0.480 & .004 \\
\hline & HIV- & 31 & & & \\
\hline \multirow[t]{2}{*}{ D-KEF-Switching } & $\mathrm{HIV+}$ & 240 & 0.138 & 0.690 & .027 \\
\hline & HIV- & 180 & & & \\
\hline \multirow[t]{2}{*}{ D-KEFS-Motor Speed } & $\mathrm{HIV}+$ & 61 & 0.229 & 0.695 & .629 \\
\hline & HIV- & 53 & & & \\
\hline
\end{tabular}

Bootstrap Hypothesis Test; Significance level $(p)$ at 0.05 .

The HIV+ group showed statistically significant poorer performance than the HIV- group in psychomotor processing $(p<.001,95 \%$ CIs $[0.030] \&[0.282])$. Overall processing speed was significantly poorer for the HIV+ group as evidenced by the following tasks: psychomotor processing, visual scan and switching. Performance in motor speed did not differ significantly for the two groups $(p=.629,95 \%$ CI [0.229] \& 
[0.695]); however, a comparison across serostatus (Table 4) shows that the HIV+ group evidenced slower motor speed relative to the HIV-group.

A pattern of poorer performance was also observed in the memory domain ( $\mathrm{p}$ $<.001,95 \%$ CIs [0.424] \& [0.518]). Variable results were observed for the attention domain, where the HIV+ group performed significantly poorer than the HIV-group in a visual search measure of attention $(p=.004,95 \%$ CIs [0.071] \& [0.480]); however performance on simple attention and complex attention tasks yielded no significant results.

Furthermore, significant group differences in performance on the visuoconstructional domain $(p<.001,95 \%$ CIs [0.732] \& [0.950]) was observed.

Table 5. Comparison of HIV- participant total DRS scores to age and education adjusted scores (Schmidt et al., 1994)

\begin{tabular}{lll}
\hline Variables & Schmidt et al. (1994) & Current study \\
\hline Educational level & $4-9$ years & $4-15$ years \\
Age & $50-59$ & $42-60$ \\
$\mathrm{~N}$ & 119 & 25 \\
Mean & 140.7 & 130.28 \\
$\mathrm{SD}$ & 4.8 & 9.6 \\
Lowest quintile & 135 & 120 \\
Lower quartile & 139 & 125 \\
Median & 142 & 132 \\
Upper quartile & 143 & 139 \\
\hline
\end{tabular}

$\mathrm{N}=$ sample size; $\mathrm{SD}=$ standard deviation

Table 5 illustrates comparative global performance on the DRS-2 by age and education group for the current study and the closest sample equivalent in the Schmidt et al. (1994) study. A one-sample t-test was conducted between the HIV-sample and the normative sample in Schmidt et al. (1994). Results indicated significantly poorer scores 
in global cognitive performance $(\mathrm{t}=5.43 ; \mathrm{p}<.001)$ in the HIV-group. Furthermore, the HIV+ group in the current sample also showed significantly poorer performance than the normative group $(\mathrm{t}=8.44 ; \mathrm{p}<.001)$.

\section{Discussion}

The results presented above advance previous findings that adults and older adults with HIV evidence significantly poorer performance in cognitive domains of psychomotor speed, memory, visuoconstruction and executive functioning when compared to seronegative adults (Fazeli et al., 2014; Sorlini et al., 2014). Global cognitive functioning in the HIV cohort was also significantly poorer than the age matched HIV-control group. Although performance profiles were lower and response speed was slower on all attention tasks for the HIV+ group when compared to the HIV-group, simple attention (DRS-2) and selective attention (Stroop Test) performance scores indicated no significant differences between the groups. These results are commensurate with findings on the cognitive profile in early stage HIV (Woods et al., 2009). In a study comparing neuropsychological functioning between a seropositive and seronegative group, Reger, Welsh, Razani, Martin and Boone (2002) observed that effect sizes pertaining to group differences on tasks of attention and concentration increased as a function of disease severity.

Of particular interest is the performance variation in significant score patterns on different attention tasks. Woods et al. (2009) and Levine et al. (2008) espoused the idea that one domain of overlap on tasks measuring attention is psychomotor speed and speed of information processing. Given that impairment in various neuropsychological domains, 
particularly in speed of mental processing, have been observed in HIV+ adults and older adults, this aspect needs consideration when interpreting performance (Vance et al., 2013). In the current study, performance on simple untimed and complex timed attention tasks without psychomotor demands (DRS-2 Attention and Stroop Test) indicated no significant differences between the groups. On the tasks of attention requiring a psychomotor interface (D-KEFS-Visual Scanning, D-KEFS-Switching and SDMT) significant group differences were observed. The results may indicate that when processing demands are augmented (divided attention), HIV associated psychomotor and cognitive functioning is attenuated (Grant, 2008). Multiple factors such as sensory modality through which the tasks are completed (visual/auditory), time pressure, psychomotor component and complexity of task demands need to be considered when specifying and measuring the attention deficit in adults and older adults aging with HIV in the earlier disease stages.

The current investigation identified significant group differences in multiple cognitive domains. However, no significant difference in underlying component motor skills between the two groups was found. Poorer performance in psychomotor speed/information processing speed may not have emerged as secondary to basic motor slowing, but rather may indicate impairment in higher order cognitive processing capabilities (Woods et al., 2009).

Deficits in executive functioning are one of the foremost features in the neuropsychological profile of HIV (Dawes, 2008; Iudicello et al., 2012; Woods et al., 2009). In this study cohort performance on specific executive functioning tasks (set shifting and graphomotoric perseverative tests) that rely on psychomotor speed may be 
partially modulated by compromised functioning in that domain. Fellows, Byrd and Morgello (2014) established that in HIV+ individuals, processing speed accounted for $77 \%$ of variability in executive functioning and appears to notably mediate the influence of age-associated changes.

Results corroborated the association between duration on anti-retroviral regimens and neuropsychological functioning that have been reported in several studies (Cross, Combrinck, \& Joska, 2013; Kahouadji et al., 2013). Duration of time on anti-retroviral therapy in an aging HIV population may potentiate the risk for CNS neurotoxicity, which can compromise cognitive integrity and result in poorer outcomes on tasks requiring speed of mental processing (timed tasks). The participants' adherence to medication use was not monitored and these results on anti-retroviral use have to be interpreted with this caveat.

According to Schmidt et al. (1994), the original MDRS shows a steady decline and a wider range of scores with decreasing educational levels. A significant, positive correlation was found between global DRS-2 scores and years of education, indicating that the DRS-2 scores in the current study parallel the same trend in range of scores. However, analyses also indicated a significantly poorer cognitive performance in the current study control group as compared to the original MDRS sample. These comparisons suggest that although the normative and control group each show a decline in cognitive performance with decreasing educational levels, caution should be applied when using cut-off score determinants to categorize severity of impairment based on norms outside the South African context. Considering the socio-demographic parameters of participants in this study, particularly with regard to the quality and quantity of 
education status, further research utilizing larger sample sizes is required to determine the clinical utility of the DRS-2 in determining the severity of impairment in adults and older adults with HIV.

There are various potential limitations to this study. The length of the neuropsychological assessment and the specificity of the cohort being assessed resulted in a small sample size. The HIV+ group comprised individuals not older than 55 and future studies should include a wider age range to account for common neurological comorbidities in an older group. Due to incomplete medical records, the provision of a clearer clinical profile of the HIV+ participants was hindered. A more detailed assessment is required of psychiatric status as a potential mediator of neuropsychological outcomes in an older HIV+ cohort.

The recruitment procedure employed may have resulted in selection bias, as the medical professionals were the first point of contact in approaching participants. However, due to the ethical parameters governing HIV participant recruitment, the main responsibility of the study was to protect the participants' right to confidentiality, anonymity and voluntary participation. Several challenges were encountered in ascertaining the HIV status of the participants. This was due to methodological aspects related to ethical issues pertaining to HIV. It is possible that some individuals in the HIV negative sample may have been HIV positive. However, due to ethical constraints, the participants' medical records were taken as the final authority in terms of sampling. The performance trend on the DRS-2 global score aligns with the focus of the current debate in the literature (Joska et al., 2011; Robbins et al., 2013) regarding the cultural 
appropriateness of using established neuropsychological test norms to determine the severity of HIV associated neuropsychological impairment in the South African context.

\section{Conclusion}

In summary, the HIV+ group performed significantly poorer in comparison to the HIVgroup on global neuropsychological functioning and specifically in cognitive domains of executive functioning, memory, psychomotor speed and visuoconstruction. Compromised cognitive status in populations aging with HIV may confer increased risk for declining functional outcomes in activities of daily living, poor medication adherence, maintenance of clinic attendance, and lower overall health-related quality of life (Jacks et al., 2015; Moore et al., 2014; Rodriguez et al., 2013). Taken together with an aging HIV+ population in South Africa, studies focusing on neuropsychological functioning are essential for delineating the specific mental health care needs of this subgroup.

\section{References}

Ances, B. M., Vaida, F., Yeh, M. J., Liang, C. L., Buxton, R. B., Letendre, S., ... Ellis, R. J. (2010). HIV infection and aging independently affect brain function as measured by functional magnetic resonance imaging. The Journal of Infectious Diseases, 201(3), 336-340.

Antinori, a., Arendt, G., Becker, J. T., Brew, B. J., Byrd, D. a., Cherner, M., ... Wojna, V. E. (2007). Updated research nosology for HIV-associated neurocognitive disorders. Neurology, 69(18), 1789-1799. 
Chernick, R. M., \& LaBudde, A. R. (2011). An introduction to bootstrap methods with applications to $R$. New Jersey: Wiley.

Cross, H.M., Combrinck, M.I., \& Joska, J.A. (2013). HIV-associated neurocognitive disorders: Antiretroviral regimen, central nervous system penetration effectiveness, and cognitive outcomes. South African Medical Journal, 103(10), 758-762.

Cysique, L. A, Maruff, P., Bain, M. P., Wright, E., \& Brew, B. J. (2011). HIV and age do not substantially interact in HIV-associated neurocognitive impairment. The Journal of Neuropsychiatry and Clinical Neurosciences, 23(1), 83-89.

Dawes, S., Suarez, P., Casey, C. Y., Cherner, M., Marcotte, T. D., Letendre, S., ... Heaton, R. K. (2008). Variable patterns of neuropsychological performance in HIV1 infection. Journal of Clinical and Experimental Neuropsychology, 30(6), 613-626.

Fazeli, P. L., Crowe, M., Ross, L. A., Wadley, V., Ball, K., \& Vance, D. E. (2014). Cognitive functioning in adults aging with HIV: A cross-sectional analysis of cognitive subtypes and influential factors. Journal of Clinical Research in HIV AIDS and Prevention, 1(4), 155-169.

Fellows, R. P., Byrd, D. A., \& Morgello, S. (2014). Effects of information processing speed on learning, memory, and executive functioning in people living with HIV/AIDS. Journal of Clinical and Experimental Neuropsychology, 36(8), 806817. 
Field, A. (2009). Discovering statistics using spss (3rd ed.). London: SAGE Publications Ltd.

Gannon, P., Khan, M. Z., \& Kolson, D. L. (2011). Current understanding of HIVassociated neurocognitive disorders pathogenesis. Current Opinion in Neurology, 24(3), 275.

Grant, I. (2008). Neurocognitive disturbances in HIV. International Review of Psychiatry, 20, 33-47.

Habib, A. G., Yakasai, A. M., Owolabi, L. F., Ibrahim, A., Habib, Z. G., Gudaji, M., ... Nashabaru, I. (2013). Neurocognitive impairment in HIV-1-infected adults in SubSaharan Africa: A systematic review and meta-analysis. International Journal of Infectious Diseases, 17(10), e820-e831.

Hardy, D. J., \& Vance, D. E. (2009). The neuropsychology of HIV/AIDS in older adults. Neuropsychology Review, 19(2), 263-272. doi:10.1007/s11065-009-9087-0

Hontelez, J. A. C., Lurie, M. N., Newell, M.-L., Bakker, R., Tanser, F., Bärnighausen, T., ... de Vlas, S. J. (2011). Ageing with HIV in South Africa. AIDS (London, England), 25(13), 1665-1667.

Iudicello, J. E., Woods, S. P., Deutsch, R., Grant, I., \& The HIV Neurobehavioral Research Program Group. (2012). Combined effects of aging and HIV infection on semantic verbal fluency: A view of the cortical hypothesis through the lens of 
clustering and switching. Journal of Clinical and Experimental Neuropsychology, 34(5), 476-488.

Jacks, A., Wainwright, D., Salazar, L., Grimes, R., York, M., Strutt, A.M., Shahani, L., Woods, S.P., \& Hasbun, R. (2015). Neurocognitive deficits increase risk of poor retention in care among older adults with newly diagnosed HIV infection. AIDS, 29, 1711-1714.

Joska, J. A., Westgarth-Taylor, J., Myer, L., Hoare, J., Thomas, K. G. F., Combrinck, M., ... Flisher, A. J. (2011). Characterization of HIV-Associated Neurocognitive Disorders among individuals starting antiretroviral therapy in South Africa. AIDS and Behavior, 15(6), 1197-1203.

Jurica, P. J., Leitten, C. L., \& Mattis, S. (2001). No Titl DRS-2 dementia rating scale-2: professional manual. Psychological Assessment Resources.

Kahouadji, Y., Dumurgier, J., Sellier, P., Lapalus, P., Delcey, V., Bergmann, J.F., Hugon, J., \& Paquet, C. (2013). Cognitive function after several years of antiretroviral therapy with stable central nervous system penetration score. HIV Medicine, 14, $311-315$.

Lawler, K., Jeremiah, K., Mosepele, M., Ratcliffe, S. J., Cherry, C., Seloilwe, E., \& Steenhoff, A. P. (2011). Neurobehavioral effects in HIV-positive individuals receiving highly active antiretroviral therapy (HAART) in Gaborone, Botswana. PLOS ONE, 6(2), 1-7. 
Levine, A. J., Hardy, D. J., Barclay, T. R., Reinhard, M. J., Cole, M. M., \& Hinkin, C. H. (2008). Elements of attention in HIV-infected adults: Evaluation of an existing model. Journal of Clinical and Experimental Neuropsychology, 30(1), 53-62.

Lezak, M., Howieson, D., Bigler, E., \& Tranel, D. (2012). \& Tranel, D.(2012). Neuropsychological assessment (5th ed.). New (5th ed.). New York: Oxford University Press.

Liner, K. J., Ro, M. J., \& Robertson, K. R. (2010). HIV, antiretroviral therapies, and the brain. Current HIV/AIDS Reports, 7(2), 85-91.

Mills, E. J., Rammohan, A., \& Awofeso, N. (2011). Ageing faster with AIDS in Africa. The Lancet, 9772 (377), 1131-1133.

Moore, R.C., Fazelli, P.L., Jeste, D.V., Moore, D.J., Grant, I., Woods, S.P., \& The HIV Neurobehavioural Research Program (HNRP) Group. (2014). Succesful cognitive aging and health-realted quality of life in younger and older adults infected with HIV. AIDS Behaviour, 18, 1186-1197.

Morgan, E. E., Woods, S. P., Delano-Wood, L., Bondi, M. W., Grant, I., \& The HIV Neurobehavioral Research Programs Group. (2011). Intra-individual variability in HIV Infection: Evidence for greater neurocognitive dispersion in older HIV seropositive adults. Neuropsychology, 25(5), 645-654.

Pallant, J. (2010). SPSS survival manual (4th ed.). Berkshire: Open University Press. 
Reger, M., Welsh, R., Razani, J., Martin, D. J., \& Boone, K. B. (2002). A meta-analysis of the neuropsychological sequelae of HIV infection. Journal of the International Neuropsychological Society: JINS, 8(3), 410-424.

Robberstad, B., \& Olsen, J. A. (2010). The health related quality of life of people living with HIV/AIDS in sub-Saharan Africa - a literature review and focus group study. Cost Effectiveness and Resource Allocation, 8(5), 1-11.

Robbins, R. N., Joska, J. A., Thomas, K. G. F., Stein, D. J., Linda, T., Mellins, C. A., Remien, R. H. (2013). Exploring the utility of the Montreal Cognitive Assessment to detect HIV-associated neurocognitive disorder: The challenge and need for culturally valid screening tests in South Africa. The Clinical Neuropsychologist, 27(3), 437-454. doi: 10.1080/13854046.2012.759627

Rodriguez-Penney, A.T., Iudicello, J.E., Riggs, P.K., Doyle, K., Ellis, R.J., Letendre, S.L., Grant,I., Woods, S.P., \& The HIV Neurobehavioural Research Program (HNRP) Group. (2013). Co-morbidities in persons infected with HIV: Increased burden with older age and negative effects on health-related quality of life. AIDS Patient Care and STDs, 27(1), 5-16.

Schmidt, R., Freidl, W., Fazekas, F., Reinhart, B., Grieshofer, P., Koch, M., ... Lechner, H. (1994). The Mattis Dementia Rating Scale: normative data from 1,001 healthy volunteers. Neurology, 44(5), 964-966. 
Scott, J. C., Woods, S. P., Carey, C. L., Weber, E., Bondi, M. W., \& Grant, I. (2011). Neurocognitive consequences of HIV infection in older adults: An evaluation of the “Cortical” hypothesis. AIDS and Behavior, 15(6), 1187-1196.

Seider, T. R., Luo, X., Gongvatana, A., Devlin, K. N., de la Monte, S. M., Chasman, J. D., ... Cohen, R. A. (2014). Verbal memory declines more rapidly with age in HIV infected versus uninfected adults. Journal of Clinical and Experimental Neuropsychology, 36(4), 37-41.

Sheppard, D.P., Woods, S.P., Bondi, M.W., Gilbert, P.E., Massman, P.J., Doyle, K.L., \& The HIV Neurobehavioural Research Program ( HNRP) Group. ( 2015). Does older age confer an increased risk of incident neurocognitive disorders among persons living with HIVdisease? The Clinical Neuropsychologist, 29(5), 656-677.

Singh, D., Joska, J. A., Goodkin, K., Lopez, E., Myer, L., Paul, R. H., ... Sunpath, H. (2010). Normative scores for a brief neuropsychological battery for the detection of HIV-associated neurocognitive disorder (HAND) among South Africans. BMC Research Notes, 3(28), 1-7. doi:10.1186/1756-0500-3-28.

Sorlini, M.L., Foca, E., Salvi, A., Compostella, S., Brianese, N., Ferraresi, A., ... Roldan, E.Q. (2014). Elderly women with Human Immunodeficiency Virus infection: Is there an effect of the virus on neuropsychological profile? Journal of the American Geriatrics Society, 62(1), 194-195. doi: 10.1111/jgs.12625 
Suarez, S. V., Stankoff, B., Conquy, L., Rosenblum, O., Seilhean, D., Arvanitakis, Z., .. . Dubois, B. (2000). Similar subcortical pattern of cognitive impairment in AIDS patients with and without dementia. European Journal of Neurology, 7(2), 151158. doi: 10.1046/j.14681331.2000.00050.x

Vance, D.E., Fazeli, P.L., \& Gakumo, C.A. (2013). The impact of neuropsychological performance on everyday functioning between older and younger adults with and without HIV. Journal of the Association of Nurses in AIDS Care, 24(2), 112-125.

Vitaliano, P.P., Breen, A.R., Russo, R., Albert, M., Vitiello, M., \& Prinz, P.N. (1984). The clinical utility of the Dementia Rating Scale for assessing Alzheimer patients. Journal of Chronic Diseases, 37(9-10), 743-753.

Wendelken, L.A., \& Valcour, V. (2012). Impact of HIV and aging on neuropsychological status. Journal of Neurovirology, 18, 256-263.

Woods, S. P., Moore, D. J., Weber, E., \& Grant, I. (2009). Cognitive neuropsychology of HIV-associated neurocognitive disorders. Neuropsychology Review, 19(2), 152-168. 